\title{
Situaciones de acción y juegos cooperativos. Un análisis teórico sobre la doble dimensión de la acción colectiva para la gobernanza comunitaria de recursos de uso común
}

\section{Mauricio García Ojeda}

Universidad de la Frontera. Departamento de Ciencias Sociales mauricio.garcia@ufrontera.cl

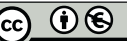

\section{Resumen}

El artículo tiene un carácter teórico y su objetivo es analizar la acción colectiva comunitaria en torno a recursos naturales de uso común (RNUC) desde un punto de vista diferente. Desde los conceptos de situación de acción y situaciones de acción adyacentes, concebimos la acción colectiva no solo como el factor que permite evitar la tragedia de los comunes, sino también como un medio estratégico que utilizan comunidades para demostrar eficacia en el uso sustentable de sus RNUC y así disponer de aportes entregados por el Estado a través de instrumentos de política pública. Esos instrumentos contienen diseños institucionales que incentivan a los usuarios a generar acción colectiva, lo que refuerza el papel de las reglas comunitarias de apropiación y provisión.

Palabras clave: comunidad; cogobernanza; reglas; redes sociales; juego de seguridad; cooperación; situaciones de acción adyacentes; política pública; diseño institucional

Abstract. Situations of action and cooperative games: A theoretical analysis on the double
dimension of collective action for community governance of common-pool resources

The objective of this theoretical article is to analyze community collective action around natural common-pool resources (NCPR) from a different point of view. In line with the concepts of action situation and adjacent action situations, we conceive collective action not only as the factor that avoids the tragedy of the commons, but also as a strategic means that communities use to demonstrate effectiveness in the sustainable use of their NCPR and hence have contributions delivered by the state through public policy instruments. The institutional designs of these instruments encourage users to generate collective action, thus reinforcing the role of community rules of appropriation and provision.

Keywords: community; co-governance; rules; social networks; assurance game; cooperation; adjacent action situations; public policy; institutional design

* Artículo producto del proyecto FONDECYT de Iniciación N¹1160456. 


\title{
Sumario
}

\author{
1. Introducción \\ 3. Situación de acción operativa, \\ situaciones de acción adyacentes y \\ 2. Situación de acción y situaciones \\ de acción adyacentes \\ la doble dimensión de la acción colectiva \\ comunitaria \\ 4. Conclusiones \\ Referencias bibliográficas
}

\section{Introducción}

En la actualidad los Estados han incrementado sus esfuerzos para apoyar procesos que realizan comunidades en torno al uso colectivo de sus recursos naturales. En este marco, organizaciones estatales de nivel nacional y local buscan promover que las comunidades usen de forma sustentable los ecosistemas. Con ese propósito, los Estados utilizan diversos instrumentos de política pública. Parte de estos instrumentos son aplicados de una forma especial, pues los Estados condicionan su entrega a que las comunidades demuestren eficacia en el uso sustentable de recursos naturales.

Entre los instrumentos aplicados desde esta lógica, se incluyen, en primer lugar, la asignación de derechos de uso, la entrega de asistencia técnica y recursos productivos en el marco de la implementación de áreas naturales de conservación forestales (Araújo, et al., 2016; Cruz et al., 2016), marinas (Gruby y Basurto, 2013), y de otro tipo de figuras de administración reguladas en los planes de manejo de recursos (Stronza, 2009). En segundo lugar, se incluyen los pagos por servicios ambientales, utilizados por los Estados como instrumentos para generar bienes públicos ambientales. Se entregan incentivos monetarios, reconociendo derechos de propiedad, dotando de infraestructura y otros servicios, siempre que las comunidades produzcan determinados resultados ambientales a partir de la gestión de sus $\mathrm{RNUC}^{1}$ (Fisher et al., 2010; Kerr, 2014; Kolinjivadi et al., 2014; Merino y Robson, 2006). En tercer lugar, se utilizan los microcréditos o microfinanzas. Son recursos monetarios entregados de forma renovable a comunidades para que ejecuten proyectos de desarrollo social, productivo o ambiental (Anderson et al., 2002; Murali, 2006). En cuarto lugar, los Estados otorgan ecocertificaciones o ecoetiquetados. Son acreditaciones o sellos verdes que garantizan la sustentabilidad de procesos productivos comunitarios desde los que se elaboran diversos tipos de productos (Foley y McCay, 2014).

1. Como detallamos más adelante, los RNUC son sistemas de recursos naturales (bosques, praderas, cuencas hidrográficas, bordes costeros marinos, entre otros sistemas de recursos) respecto a los cuales existe un régimen de propiedad común. Esto permite que los integrantes de una comunidad portadora de derechos de uso accedan a unidades de recursos (número de árboles, kilos de forraje, metros cúbicos de agua, kilos de pescado, por ejemplo) de un sistema de recursos. 
A partir del análisis de la naturaleza de estos instrumentos, planteamos que enmarcan la acción del Estado hacia las comunidades en una lógica contractual y de cooperación condicional. Por una parte, las comunidades deben establecer compromisos explícitos y vinculantes a través de contratos, planes de gestión de recursos, convenios de uso, etcétera, y por otra, deben rendir cuentas del efectivo cumplimiento de esos compromisos. Así logran acceder de forma continua a aportes estatales que consideran fundamentales, pues mejoran la condición de sus integrantes, ya que hacen posible que se beneficien en el presente y en el futuro de sus RNUC. Sin embargo, para que la colaboración estatal sea realmente una oportunidad, las comunidades requieren, como indicamos, cumplir compromisos, lo que, a su vez, implica que deben ser capaces de generar la acción colectiva necesaria para obtener resultados en términos de sustentabilidad ambiental.

Considerando la lógica contractual y de cooperación condicional señalada, en este artículo proponemos una perspectiva teórica para abordar la acción colectiva desde un prisma diferente. Desde los conceptos de situación de acción y situaciones de acción adyacentes, desarrollados a partir de Understanding institutional diversity de Ostrom (2005), visualizamos la acción colectiva no solo como un fin en sí mismo para evitar la sobreexplotación de RNUC, sino, además, como un medio estratégico que utilizan las comunidades para lograr usar de forma sustentable sus RNUC y así acceder a instrumentos de política pública como los señalados.

En este sentido, planteamos que los instrumentos de política pública contienen diseños institucionales, es decir, un conjunto de reglas que incentivan a los integrantes de comunidades para lograr la acción colectiva, lo que permite que cuenten con derechos, servicios y bienes provistos desde el Estado. Por esta razón, se produce una adyacencia o conexión entre la situación de acción operativa (la producida entre los usuarios de un sistema de recursos a nivel comunitario) y la situación de acción estatal, formada en el marco de la implementación de instrumentos de políticas públicas destinadas a los usuarios de RNUC. La adyacencia se produce porque el diseño institucional de los instrumentos de política afecta la lógica interna comunitaria en torno al uso de RNUC.

Esta perspectiva no ha sido abordada en la literatura sobre gobernanza de RNUC. En ese campo de conocimiento, se ha analizado la relación entre el Estado y comunidades, especialmente a partir del estudio de la cogobernanza. No obstante, no se ha estudiado específicamente esa relación como cooperación condicional mediada por instrumentos de política pública. Tampoco se ha utilizado un enfoque teórico en base a los conceptos de situación de acción y situaciones de acción adyacentes para analizar una temática como la que delimitamos en este artículo. El concepto de situación de acción ha sido utilizado para analizar procesos de gobernanza en comunidades. Por su parte, el concepto de situaciones de acción adyacentes solo ha sido aplicado de forma genérica al estudio de la gobernanza policéntrica, es decir, a procesos de coordinación entre diversos actores (comunidades, organizaciones del 
Estado, empresas, entre otras) orientados a afrontar problemas ambientales en sistemas socioecológicos.

Por lo anterior, analizar la conexión entre situaciones de acción en torno a procesos de acción colectiva creemos que aporta a la comprensión de dinámicas y resultados en entornos complejos de políticas públicas operacionalizadas en procesos de cogobernanza y gobernanza policéntrica. Considerando lo propuesto en este artículo, sus secciones se estructuran de la siguiente forma. En primer lugar, exponemos los conceptos de situación de acción y situaciones de acción adyacentes, en base a los cuales, en segundo lugar, damos a conocer la propuesta orientada a comprender la doble dimensión de la acción colectiva en torno a los RNUC. Finalmente, exponemos algunas conclusiones.

\section{Situación de acción y situaciones de acción adyacentes}

\subsection{Situación de acción}

El concepto de situación de acción fue desarrollado por Ostrom y sus colaboradores en el contexto del esfuerzo para ampliar el marco analítico inicialmente expuesto en Governing the commons. The evolution of institutions for collective action (Ostrom, 1990). Con el propósito de contar con un enfoque teórico general que incluya de forma relacionada tres niveles de análisis, que son los comportamientos individuales, las situaciones microsociales que afectan las decisiones individuales y sus resultados en dilemas sociales y el contexto socioecológico más amplio, en Understanding institutional diversity (Ostrom, 2005), se desarrolló el marco de análisis y desarrollo institucional (ADI).

En el ADI, el elemento fundamental para el análisis microsituacional es la situación de acción, que es una abstracción o generalización de los elementos esenciales de un espacio social de decisiones. En este espacio, actores (comunidades, empresas, agencias gubernamentales) con diversas preferencias interactúan y se influyen mutuamente en torno a acciones potenciales expresadas en estrategias, que, en conjunto, producen resultados que incluyen el intercambio de bienes, la competencia y/o la resolución de problemas. Cuando individuos interactúan en un contexto social con estas características, están en una situación de acción (Ostrom y Cox, 2010). De forma más específica, Ostrom (2005) plantea que las situaciones de acción en las que usuarios de RNUC se implican en tareas de aprovisionamiento, producción, distribución y apropiación se clasifican como situaciones de acción operativas.

Los elementos constituyentes de una situación de acción son: a) el conjunto de participantes (determinado número de unidades sociales individuales o colectivas con estatus y otros atributos y que son capaces de tomar decisiones); b) la posición que ocupan (relacionada con sus roles y que vinculan a los participantes con sus acciones); c) los potenciales resultados de sus acciones (que constituyen oportunidades y que son valorados en términos de resultados materiales, beneficios o costos asignados a acciones y resultados); d) las acciones disponibles (que constituyen el medio que tienen los participantes para obtener los resultados buscados en una situación de acción); e) el control ejercido 
sobre las elecciones (que varía y configura el poder sobre la concreción de las oportunidades); f) la información completa o incompleta sobre los elementos de la situación de acción en que se sitúan y de otros elementos contextuales; g) los costos y beneficios relativos a acciones y resultados (estimados con relación a los recursos iniciales disponibles y otros criterios de evaluación en torno a los resultados potenciales) (Ostrom, 2005; Ostrom, et al., 2017).

Los elementos de una situación de acción afectan la formación de preferencias, las estrategias y acciones de los actores (Poteete et al., 2010). Analizadas de forma dinámica, y en términos de la teoría de juegos, las situaciones de acción iteradas afectan sus componentes. Por ejemplo, afectan la disponibilidad de información sobre el tipo de los otros jugadores, es decir, sobre sus estrategias y valoración de costos y beneficios en una situación de acción. También las rondas repetidas afectan las acciones disponibles (estrategias) y posibles resultados, lo que puede modificar un equilibrio no cooperativo por uno cooperativo, gracias a la generación de aprendizaje social en torno a la confianza y reciprocidad. Producto del cambio de equilibrio, se modifican los resultados, con el aumento de los beneficios de los participantes implicados en una situación de acción.

\subsection{Situaciones de acción adyacentes}

Ostrom (2005) plantea que además de analizar en específico cada situación de acción, es necesario también analizar las conexiones que se producen entre ellas. Señala que los ámbitos políticos y económicos operan en órdenes policéntricos, es decir, están conformados por la coexistencia de diversos actores que operan como centros o unidades de toma de decisiones. Los actores que forman parte de situaciones de acción diferentes, al relacionarse, se influyen mutuamente o uno influye sobre el otro a través de componentes de la situación de acción a la que pertenece.

A partir del concepto de situación de acción y en base a reelaboraciones del marco AID, McGinnis (2011a, 2017) desarrolló el concepto de situaciones de acción adyacentes para analizar interacciones entre diferentes actores implicados en procesos policéntricos de gobernanza de RNUC y/o de implementación de políticas públicas. Una idea fundamental es que para explicar la estructura y resultados de equilibrio de un juego y el sentido de las decisiones de un jugador en ese juego, hay que considerar la trama completa de juegos en las que está involucrado (McGinnis, 1999, 2000). Las situaciones de acción operan de forma simultánea o secuencial y son adyacentes (están conectadas directamente) cuando los resultados de una influyen en el estado de uno o más componentes de la otra (Cárdenas et al., 2011; McGinnis, 2011b). A partir de la adyacencia entre situaciones de acción, se configuran redes de situaciones de acción adyacentes, donde las situaciones de acción son los nodos y las influencias producidas son las conexiones.

Se distinguen tres tipos de conexiones. El primer tipo corresponde a las conexiones estructurales, producidas por la influencia entre reglas de diferentes niveles de jerarquía. Por ejemplo, una regla de nivel constitucional afecta la 
situación de acción operativa que se produce en el nivel comunitario, en la que usuarios toman decisiones, traducidas en acciones que generan determinados resultados respecto a sus RNUC. La conceptualización de las conexiones estructurales se origina a partir del análisis realizado por Ostrom (2005) sobre los efectos del contexto policéntrico en la gobernanza comunitaria. La autora plantea que las situaciones de acción operativas están anidadas en conjuntos de reglas de niveles institucionales jerárquicamente mayores. Estas reglas pueden afectar diferentes elementos de la situación de acción operativa, como sus reglas, costos y beneficios de los participantes y los resultados producidos.

El segundo tipo de conexión entre situaciones de acción es la conexión de proceso, que se forma cuando el flujo de recursos (información, bienes, servicios) de una situación de acción afecta elementos de otra. El tercer tipo de conexiones son las funcionales, generadas cuando se concatenan etapas en la implementación de determinadas tareas. Cada tarea (financiamiento, manutención o provisión, coordinación, resolución de conflictos, creación de reglas, monitoreo y sanción, entre otras) implica decisiones y acciones de actores y por eso constituye una situación de acción. Entonces, dos situaciones de acción son adyacentes funcionalmente si el resultado de una situación de acción aporta al producido en otra (McGinnis, 2011a).

Desde el concepto de redes de situaciones de acción adyacentes se han estudiado diversos procesos de gobernanza policéntrica. Entre estos procesos se cuentan la coordinación entre actores para usar de forma sustentable cuencas hidrográficas en Colombia (Cárdenas et al., 2011; Cárdenas y Ortiz, 2018) y espacios costeros para la pesca de langosta en Estados Unidos (McGinnis, 2011a). También se han analizado las coordinaciones para producir electricidad desde la operación de sistemas de irrigación en la India (Kimmich, 2013) y para el desarrollo de infraestructura generadora de energía en la región del mar Báltico (Gritsenko, 2018).

Hasta aquí hemos expuesto antecedentes para caracterizar los conceptos de situación de acción y situaciones de acción adyacentes. Estos conceptos son la base de la propuesta teórica que exponemos en la siguiente sección, que permitirá comprender la doble dimensión que adquiere la acción colectiva para las comunidades usuarias de RNUC.

\section{Situación de acción operativa, situaciones de acción adyacentes y la doble dimensión de la acción colectiva comunitaria}

La idea central que planteamos en nuestra propuesta teórica es que elementos de la situación de acción operativa son afectados por el diseño institucional de instrumentos de política pública. Se produce una adyacencia entre la situación de acción estatal y la situación de acción operativa, porque el diseño institucional de los instrumentos de política pública contiene incentivos que afectan fundamentalmente los costos y beneficios de la situación de acción operativa. Por esta razón, el efecto de las reglas comunitarias que motiva a los usuarios de RNUC a cooperar en tareas de apropiación y de provisión es reforzado 
por reglas contenidas en instrumentos de política pública. Esto se debe a que las reglas indican que los instrumentos estarán disponibles para los usuarios siempre que demuestren un uso sustentable de sus RNUC.

Producto de lo anterior, la acción colectiva adquiere una doble dimensión para los usuarios de este tipo de recursos. Para desarrollar esta idea, en primer lugar, caracterizaremos la situación de acción operativa. Especificaremos los problemas de cooperación que tienen los usuarios de RNUC y por qué las reglas aportan a solucionarlos. Esto es importante para entender la lógica de la cooperación que se produce en el interior de la situación de acción operativa. Una vez caracterizada esa situación de acción, hacemos lo propio con la estatal. Por último, especificaremos cómo se produce la adyacencia entre ambas, y de esta forma aportamos elementos para comprender la doble dimensión de la acción colectiva para la gobernanza comunitaria de RNUC.

\subsection{La situación de acción operativa}

La situación de acción operativa está constituida por la interacción estratégica entre usuarios dedicados a lograr la gobernanza de sus RNUC. Con ese propósito, se implican en dos tareas que constituyen situaciones de acción específicas: a) tareas de apropiación (acceso a un sistema de recursos y de extracción de unidades de recursos); b) tareas de provisión o de cuidado y manutención del sistema de recursos. Es fundamental que las comunidades sean eficaces en ambas tareas, lo que no está asegurado, debido a la naturaleza de sus RNUC, que tienen tres propiedades distintivas: su régimen de propiedad común (colectivo o comunitario), la alta dificultad de exclusión de usuarios y la alta substractibilidad de unidades de recursos.

Un régimen de propiedad común significa que existe un grupo de individuos que comparten cinco derechos de propiedad operacionales, que son: a) acceso o entrada a un sistema de recursos; b) extracción de unidades de recursos; c) administración de formas de uso; d) exclusión, determinando quién accede al sistema de recursos; e) alienación, referido a cómo transferir cualquiera de los derechos anteriores (Ostrom y Hess, 2010; Ostrom y Schlager, 1996).

$\mathrm{Si}$, como planteaba Hardin (2009), el régimen de propiedad de recursos es inexistente y hay ausencia de derechos, los recursos naturales son de libre acceso y su vulnerabilidad es evidente, todos los usuarios podrían acceder a un sistema de recursos y nadie podría excluir a otros. Además, si cada usuario busca maximizar su bienestar consumiendo más unidades de un recurso, esperando que los otros realicen un consumo moderado, el nivel agregado de uso de un sistema de recursos disminuirá su regeneración. Este hecho afectará el flujo agregado de beneficios que provee y se reducirá el beneficio promedio neto de los usuarios. Como efecto perverso no esperado, el recurso será sobreexplotado, lo que afectará la posibilidad de utilizarlo en el largo plazo (Janssen, 2013). Así, la búsqueda unilateral del interés propio generará la tragedia colectiva.

A pesar de que los RNUC estén regulados por un régimen de propiedad colectiva, son vulnerables, porque podrían ser sobreexplotados (Ostrom, 2002). 
La razón se debe, en parte, al segundo atributo de los RNUC, que es la alta dificultad de exclusión de usuarios. Es costoso excluir del acceso al sistema de recursos y de la extracción de unidades de recursos a quienes tienen derechos operacionales y se comportan de forma oportunista o como free-rider. Estos usuarios no contribuyen a la provisión del sistema de recursos (manejo del recurso, a través de la construcción, reparación o restauración y renovación) (Ostrom, 2005). Aquí el problema de acción colectiva es un problema de provisión, expresado en la no cooperación para asumir costos relacionados con la manutención del sistema de recursos (Gardner et al., 1990).

También los RNUC son vulnerables porque son de alta substractibilidad. Este atributo se relaciona con el sistema de recursos y las unidades de recursos como factores de un sistema socioecológico que afectan una situación de acción operativa. Existe alta substractibilidad si el grado de extracción de unidades de un sistema de recursos de parte de un usuario limita o impide que unidades del recurso estén disponibles simultáneamente para otros, es decir, se sustrae o resta flujos aprovechables para el resto (Polski y Ostrom, 2017). Debido a la incertidumbre sobre la intensidad de la disponibilidad de unidades de recursos, los usuarios tienen incentivos para extraer más unidades, lo que podría destruir la capacidad del sistema de recursos para producirlas. Aquí el problema de acción colectiva es un problema de apropiación, entendido como la no cooperación respecto a la apropiación de cantidades fijas de unidades del recurso y respecto a las distribuciones espaciales y temporales de apropiación de unidades (Gardner et al., 1990).

La lógica configurada por el uso de RNUC con esas características incapacita a los usuarios para resolver problemas actuando de forma colectiva en tareas de apropiación y provisión. El problema de acción colectiva así caracterizado ha sido analizado como un problema de cooperación desde la teoría de juegos. Más específicamente, los problemas de apropiación y provisión son concebidos como dilemas sociales, referidos a situaciones en las cuales individuos orientados a maximizar sus beneficios en el corto plazo toman decisiones de forma independiente y simultánea. En ausencia de un actor externo que los coordine, empeora la situación de todos, lo que no es previsto por nadie (Ostrom, 2007). Este resultado subóptimo ha sido analizado como un equilibrio de Nash en un dilema del prisionero de una sola ronda, en el que los jugadores interactúan solo una vez, no se comunican entre sí, lo que imposibilita que establezcan acuerdos de beneficio mutuo.

Ostrom (1990) reformula el problema y modela el juego de Hardin de los pastores o juego del dilema de los comunes. La autora propone como solución alternativa que los pastores se comunican entre sí, negocian condiciones de pastoreo y establecen acuerdos vinculantes fundados en compromisos de que optarán por la estrategia cooperativa, logrando usar sustentablemente la pradera. Lo anterior implica, como un aspecto crucial, que el replanteamiento del problema de acción colectiva en torno a RNUC se basa en concebir el problema de la cooperación como un problema de coordinación que puede enfrentarse a través de la convergencia de expectativas entre los jugadores. 
Dicho en otros términos, la acción colectiva en torno a la apropiación y provisión será posible si los usuarios logran coordinar sus estrategias a través de acuerdos vinculantes y compromisos articulados a partir del interés común de encontrar una solución que, al atender los intereses colectivos, cuide los intereses particulares. En términos teóricos, se reformuló el análisis del problema de cooperación asumiendo que no se trata de un dilema del prisionero de una sola ronda. Los usuarios juegan, por el contrario, un juego de seguridad secuencial o repetido con $N$ jugadores (Cole y Grossman, 2010; Ostrom et al., 1994). En este juego se requiere que cada usuario aporte a la generación de la acción colectiva. Frente a una interacción estratégica, es necesario que los individuos tengan claro que aporta más a su beneficio en el corto y largo plazo cooperar con otros y que el desafío para hacer que la cooperación sea estable es lograr una suficiente coordinación. Si lo consiguen, entonces, la primera preferencia de cada jugador será cooperar, a diferencia del dilema del prisionero, donde la estrategia dominante era no cooperar, dadas las preferencias de los jugadores.

En un juego de seguridad, el orden de preferencias de $A$ y $B$ es $\mathrm{CC}=4>\mathrm{DC}=3>\mathrm{DD}=2>\mathrm{CD}=1$. Esto quiere decir, en primer lugar, que para ambos jugadores su primera preferencia es cooperar y que el otro coopere, por lo que obtienen su mayor pago, que es un 4 . En segundo lugar, prefieren que no se abuse de la cooperación que ofrecen, lo que se recompensa con un pago de 3. En tercer orden, prefieren la no cooperación mutua, con un pago 2 y el peor resultado para ambos es cooperar y que el otro jugador no coopere, por lo que obtienen la utilidad más baja, que es 1 . En este juego hay dos equilibrios de Nash en estrategias puras: cooperar-cooperar (CC) y no cooperar-no cooperar (DD), dado que no hay una estrategia estrictamente dominante. Cada jugador es un cooperador condicional, es decir, inicialmente prefiere cooperar y valora la cooperación del otro, pero cooperará siempre y cuando el otro jugador también lo haga. Si ambos jugadores tienen la creencia de que el otro prefiere cooperar a no cooperar, cooperarán y se producirá el equilibrio (CC), que es un óptimo de Pareto.

No obstante, es un escenario de información incompleta, si $A$ y $B$ no tienen la seguridad o certeza respecto a las preferencias del otro, pueden optar por reducir los riesgos en términos de reducir la posibilidad de encontrarse en un escenario que signifique internalizar pérdidas (cooperar y que el otro no coopere, que es su peor pago). Por esta razón, ambos jugadores podrían optar por no cooperar, produciéndose el equilibrio de no cooperación bilateral (DD, que reporta a $A$ y $B$ pagos inferiores a los obtenidos en CC). Como se advertirá, en el juego de seguridad las expectativas de un jugador respecto al otro jugador son determinantes para el surgimiento de la cooperación. Si $A$ y $B$ tienen: a) creencias de que el otro jugador tiene preferencias propias de un juego de seguridad; b) creencias de que el otro jugador tiene creencias de que él tiene preferencias asociadas a la estructura de pagos de ese juego, podrán coordinarse para lograr un equilibrio cooperativo (Herreros, 2007).

En el juego de seguridad secuencial con $N$ jugadores, el equilibrio cooperativo es un equilibrio frágil. Esto se debe a que se requiere que cada individuo 
aporte a la generación de la acción colectiva. Para que los aportes se produzcan, es necesario reducir la incertidumbre respecto a la adscripción efectiva a la estrategia cooperativa de parte de todos los jugadores, de tal forma que sea de conocimiento común de que en los hechos eso ocurre (Gupta, 2005). La interrogante en torno al problema o fallo de coordinación ha sido, por tanto, ¿cómo reducir la incertidumbre de cada jugador respecto a las estrategias de los otros? La respuesta a esta interrogante permite afrontar el problema sobre ¿Cómo lograr que los jugadores se orienten hacia el equilibrio cooperativo y que este equilibrio sea estable, de forma que se obtenga un resultado Pareto superior sostenido? (Bowles, 2004).

La respuesta ofrecida por Ostrom y sus colaboradores se ajustó a los fundamentos de la nueva economía institucional y se refiere a la importancia de las reglas operativas de apropiación y provisión que utilizan los usuarios de RNUC para afrontar los problemas de apropiación y provisión. Las reglas de apropiación especifican los usuarios que pueden acceder al sistema de recursos, la extensión y límites del sistema de recursos y espacios físicos o localización para su uso. También especifican el tipo de recursos extraíbles, la temporalidad de acceso al sistema de recursos, las magnitudes de extracción de unidades de recursos y las asignaciones de uso - espacial, temporal, cantidades, usuarios, tipo de recursos - según condiciones climáticas, de nivel de abundancia o escasez por movilidad del recurso, etcétera.

Las reglas de apropiación incorporan los derechos de acceso, extracción y de exclusión antes señalados. Las reglas de provisión prescriben condiciones sobre el cuidado, la manutención o la renovación de un sistema de recursos. Establecen obligaciones de provisión expresadas en contribuciones (cuotas, tiempo de trabajo, etcétera), que implican costos para los usuarios. Es necesario que estas reglas sean congruentes con las de apropiación, para cuidar la proporcionalidad entre beneficios derivados del acceso a unidades de recursos y costos implicados en la provisión del sistema de recursos. En complemento, las reglas de supervisión prescriben condiciones de cumplimiento de reglas de apropiación y provisión, específicamente, indican quién supervisa y sanciona y dónde, cómo y cuándo lo hace.

Las reglas inciden directamente en un elemento de una situación de acción operativa que son los costos y beneficios, los que en un juego de estrategia se expresan en los pagos. Al afectar los costos y beneficios, las reglas afectan dos elementos adicionales de una situación operativa, que son el set de acciones disponibles, es decir, las estrategias y los posibles resultados, es decir, los equilibrios del juego.

Con relación a los costos y beneficios, como señalan Cox et al. (2013), las reglas establecen beneficios derivados de la apropiación y costes relacionados con la provisión. Respecto a los beneficios, bajo un régimen de propiedad común, los derechos de propiedad de acceso, extracción y exclusión, además de las reglas de apropiación, otorgan derechos a los usuarios para disponer de unidades de un recurso. Por esta razón, los usuarios forman creencias que otorgan un nivel de certeza sobre beneficios futuros que obtendrán produc- 
to de la distribución equitativa de unidades de recursos. Por ejemplo, en la gestión comunitaria de canales de irrigación, los derechos de extracción y las reglas de acceso configuran pagos de los usuarios, porque establecen los beneficios esperados (en un período anual, por ejemplo) en sus actividades agrícolas, gracias a la disponibilidad de agua especificada en unidades del recurso (Ostrom, 1995).

Para entender cómo las reglas inciden en los costos, señalamos que un régimen de propiedad colectivo incluye derechos de administración, que implican que los usuarios de RNUC deben asumir deberes de provisión de un sistema de recursos. Con relación al ejemplo de los regantes, las reglas de provisión negociadas hacen que los usuarios asuman costos de manutención del sistema de recursos, traducidas en trabajo físico y financiamiento para la dotación de infraestructura y actividades para su cuidado (revestimiento, limpieza, etcétera). Estos costos se expresan en pagos que se restan de las ganancias, generándose una utilidad neta que, si es mayor que la situación de statu quo (de lo que provee el canal sin hacer mejoras) y es mayor a los costes de provisión, generará incentivos para asumir los costos. La razón es que esos costos son considerados por los usuarios como inversiones en capital físico que viabilizan el suministro de beneficios que otorga el sistema de recursos. Gracias a esa disposición de asumir costos, se incrementa la productividad agrícola expresada en unidades cultivadas (Ostrom, 1995).

Las reglas son cursos de acción coordinados que, al afectar la estructura de una situación de acción, incrementan las ganancias de todos los usuarios (Gardner et al., 1990). Así, cada usuario tiene la creencia de que siguiendo estrategias cooperativas, al cumplir reglas de apropiación y provisión, no será explotado por otros, pues nadie tendría incentivos en comportarse como un free-rider. Si la asignación y la provisión de RNUC es más previsible gracias a las reglas, es posible afrontar problemas de apropiación y provisión, porque los usuarios reducen la incertidumbre seleccionando endógenamente un equilibrio cooperativo en un juego de seguridad ${ }^{2}$.

2. Es importante señalar que en la literatura sobre gobernanza de RNUC se enfatiza la importancia que tienen las redes sociales comunitarias, en complemento con las reglas de apropiación, provisión y sanción, para generar condiciones que favorecen el surgimiento y la estabilidad de un equilibrio cooperativo en un juego de seguridad iterado. Los juegos repetidos están enraizados en redes sociales comunitarias que, por una parte, tienen una topología propia de las redes de mundo pequeño — small world—. Son redes con un promedio de baja distancia entre cualquier par de nodos, generadas por una alta clusterización y porque pocos nodos operan como intermediarios. Dada su naturaleza, ese tipo de redes favorece la circulación de información sobre el comportamiento de sus integrantes de forma rápida y homogénea hacia todas las regiones de la red. Por esta razón, el comportamiento de cada integrante de la red es altamente observable para el resto. Los usuarios de RNUC así tienen incentivos para orientarse hacia el cumplimiento de reglas, lo que, a su vez, permite la formación de confianza necesaria para que los usuarios sitúen sus interacciones en un juego de seguridad, seleccionando el equilibrio cooperativo. Sobre las redes de mundo pequeño y mecanismos de influencia social, ver Linares (2015). 


\subsection{Situación de acción estatal}

Analizamos brevemente la situación de acción estatal porque no es el foco de atención fundamental. Esta situación de acción se produce en torno a la tarea que realizan organizaciones del Estado destinadas a implementar instrumentos de política pública condicionales que buscan aportar a los procesos de gobernanza comunitaria. Esta tarea también constituye una situación de acción, pues se despliega desde interacciones estratégicas relativas a tareas secuenciales y desde niveles decisionales diferentes. En este sentido, al igual que en el caso de la situación de acción operacional, la que implica al Estado se puede diseccionar en varias situaciones de acción con conexiones funcionales que forman una red de situaciones de acción adyacentes. Las interacciones estratégicas se producen también porque el Estado requiere solucionar problemas de agencia derivados de sucesivas delegaciones principal-agente al interior de su organización jerárquica (Lapuente, 2007). Este asunto es crítico porque los problemas principal-agente como dilemas sociales en la burocracia pública afectan la calidad de las prestaciones entregadas a comunidades usuarias de RNUC (Ostrom et al., 1994).

\subsection{Adyacencia entre las situaciones de acción operativa y estatal}

Como una reformulación del marco AID, Ostrom (2007) propone y luego refina junto a sus colaboradores (Basurto et al., 2013; Ostrom, 2009, 2011; McGinnis y Ostrom, 2014) el marco de análisis de sistemas socioecológicos. Este marco establece que las interacciones entre los elementos de una situación de acción y sus resultados son afectados por factores externos, específicamente por sistemas de recursos, unidades de recursos, sistemas de gestión y por el contexto político, económico y social. Los sistemas de recursos y las unidades de recursos afectan una situación de acción operativa, específicamente sus resultados, porque los problemas generados por la alta substractibilidad y la dificultad de exclusión de los RNUC podrían generar una tragedia de los comunes.

No obstante, para evitar esa tragedia, la situación de acción operativa también puede afectarse positivamente por el sistema de gestión ${ }^{3}$, específicamente por los derechos de propiedad relacionados con el régimen de propiedad comunitario. Los derechos delimitan las condiciones de uso del sistema de recurso y hacen posible la existencia de reglas de apropiación y provisión, que, como indicamos, favorecen el surgimiento de la acción colectiva comunitaria.

3. Un sistema de gestión, que es uno de los elementos del marco de análisis de sistemas socioecológicos, se refiere a interacciones recurrentes, que constituyen formas organizativas que inciden en los usuarios que las configuran y en el sistema de recursos (McGinnis y Ostrom, 2014). Según Poteete et al. (2010), un sistema de gestión está conformado por organizaciones gubernamentales, organizaciones no gubernamentales, estructuras de redes entre comunidades y organizaciones gubernamentales y no gubernamentales, sistemas de derechos de propiedad, reglas operativas (comunitarias), reglas de elección colectiva, reglas constitucionales y por el proceso de monitoreo y sanción comunitario. 
En complemento, la situación de acción operativa está positivamente influida por el contexto político, económico y social ${ }^{4}$, específicamente por el contexto político, en cuyo marco se diseñan e implementan políticas gubernamentales de recursos. Los instrumentos de política pública condicionales, como las prestaciones en torno a la implementación de áreas naturales protegidas, los pagos por servicios ambientales, los microcréditos, las concesiones de uso de recursos naturales estatales y las ecocertificaciones, son, por tanto, un tipo especial de políticas gubernamentales de recursos. Son instrumentos generados desde la situación de acción estatal y afectan la situación de acción operativa, por lo que producen adyacencia entre ambas.

Sobre esa adyacencia, tenemos como punto de partida lo señalado por Cole y McGinnis (2017): las intervenciones estatales para implementar políticas afectan el funcionamiento de elementos de la situación de acción de usuarios de RNUC y sus resultados. Pero ¿en qué sentido la implementación de instrumentos de política antes señalados afectan la situación de acción operativa? Considerando lo antes indicado sobre los tipos de conexiones que hacen adyacentes dos situaciones de acción, que son la estructural, de proceso y funcional, proponemos que se producen esos tres tipos de conexiones.

En primer lugar, la conexión es estructural, debido a que los instrumentos de política pública condicionales contienen diseños institucionales, es decir, un conjunto de reglas formales que producen incentivos que afectan la situación de acción operativa. O sea, una regla de nivel nacional tiene consecuencias en el nivel operativo de toma de decisiones sobre el uso de RNUC. En segundo lugar, la conexión es de proceso, porque a través de los instrumentos de política pública se transfieren recursos que afectan elementos de la situación de acción operativa. En tercer lugar, la conexión es funcional, porque un instrumento de política pública expresado en recursos o servicios entregados a una comunidad es resultado de la situación de acción estatal y ese resultado afecta elementos de la situación de acción operativa, específicamente resultados expresados en el uso sustentable de sus RNUC.

Entre esos tipos de conexiones centramos el análisis en la conexión estructural, porque en nuestra propuesta teórica señalamos que los instrumentos de política pública que nos interesan contienen un diseño institucional que genera incentivos que afectan elementos de la situación de acción operativa. Para identificar y caracterizar teóricamente las características del diseño institucional de los instrumentos de política pública, indicamos que si bien son diferentes porque algunos se refieren a concesiones de derechos de uso, otros a financiamientos y otros a certificaciones, tienen en común que son condicionales a la formalización de compromisos sobre resultados y a la demostración de logros respecto a esos resultados.

4. El contexto político, económico y social incluye las siguientes variables contextuales: desarrollo económico, tendencias demográficas, estabilidad política, tecnología, políticas gubernamentales de recursos, incentivos de mercado y organización de los medios (Poteete et al., 2010) 
En el caso de las concesiones de derechos de uso en áreas de conservación y otras formas de administración territorial, el Estado entrega derechos de propiedad (de acceso, extracción, administración y exclusión). Lo hace bajo la condición de que las comunidades formalicen planes de manejo (de gestión o de gobernanza), que establecen formas de uso de un sistema de recurso (quién, cuándo y cómo los usuarios participarán en actividades de extracción y provisión en un sistema de recursos). Los planes de manejo especifican compromisos sobre los cuales los usuarios deben informar de los grados de cumplimiento, y según la evaluación que se realice al respecto, el Estado renueva la concesión de derecho de uso por un nuevo período de tiempo5.

Los financiamientos, como otro tipo de aporte estatal condicional, están constituidos por los pagos por servicios ambientales y los microcréditos. Ilustrando con los pagos por servicios ambientales, los usuarios de RNUC y el Estado formalizan contratos o convenios donde establecen condiciones de la compensación por el servicio ecosistémico o de conservación entregado (área, régimen de propiedad, tipo de servicio aportado, obligaciones en torno al servicio, condiciones de pago, entre otras). En este marco, se realizan monitoreos y evaluaciones de desempeño, a través de informes e inspecciones de campo, que entregan información sobre el grado de cumplimiento, lo que es útil para decidir la continuidad de los contratos ${ }^{6}$. En el caso de las eco-certificaciones, se especifican estándares de sustentabilidad de procesos productivos asociados al uso de sistemas de recursos. Luego, se establecen acuerdos formales, y su cumplimiento es evaluado por agencias estatales o agencias auditoras. La renovación de la certificación está condicionada al cumplimiento de los estándares de los esquemas de certificación estatales 7 .

5. Por ejemplo, en Chile comunidades indígenas solicitaron al Estado la concesión de Espacios Costeros Marinos de Pueblos Originarios (ECMPO), que es un área de borde costero que puede usar, una vez aprobado, un plan de administración y un plan de manejo. En estos documentos se especifica cómo se realizará la conservación de los recursos y el medioambiente, a través de un programa de explotación que detalla resultados esperados y una propuesta metodológica para el seguimiento y la evaluación del desempeño del plan. Además, es necesario que se produzca la formalización de un convenio de uso. Si se aprueban los informes de actividades a través de los cuales organizaciones públicas realizan el monitoreo y seguimiento de los planes de manejo, la concesión de uso del ECMPO es de carácter indefinido (República de Chile, 2008).

6. Por ejemplo, en Brasil, el gobierno federal impulsó el Programa Proambiente, con el fin de promover la provisión de servicios hídricos de parte de comunidades de la Amazonía. En este marco, se firmaron acuerdos comunitarios, y en planes de utilización de recursos se establecieron resultados específicos de la gestión comunitaria, relacionados con la recuperación de las funciones hidrológicas de los ecosistemas, la conservación de los suelos y el desarrollo de prácticas de agricultura sostenible. La monitorización se realizó a través de informes mensuales en los que se verificó el cumplimiento de metas y de auditorías de actividades. Además, cada seis meses se realizaron evaluaciones de proceso, que, si eran positivas, permitían la continuidad contractual. En caso contrario, se disminuyeron o cesaron los pagos (Greiber, 2010).

7. En ese marco han operado, por ejemplo, las ecocertificaciones impulsadas por el gobierno de Estados Unidos junto a organizaciones privadas para fomentar la extracción sustentable en pequeñas pesquerías en Baja California (Foley y McCay, 2014). 
La condicionalidad respecto a la formalización y el cumplimiento de compromisos constituye la base del funcionamiento de este tipo de instrumentos. Desde una perspectiva teórica, proponemos que su diseño institucional tiene tres atributos que refuerzan la motivación de los usuarios para lograr la acción colectiva y usar de forma sustentable sus RNUC. Estos atributos son: formalización ex-ante de compromisos creíbles, rendición de cuentas ex-dure y ex-post, y renovación condicional ex-post como sanción positiva creíble. A continuación, nos referimos a cada una de esos atributos.

a) Formalización ex-ante de compromisos creíbles. Desde la perspectiva temporal de un contrato, los usuarios de RNUC y el Estado, como partes implicadas, acuerdan y explicitan compromisos mutuos. Respecto a los usuarios de RNUC, los compromisos se refieren a procesos y resultados relacionados con el uso sustentable de sistemas de recursos. Desde el Estado, el compromiso es entregar los beneficios contenidos en un instrumento de política pública, siempre que tenga conformidad del cumplimiento de los compromisos de los usuarios.

Teniendo la creencia de que el compromiso del Estado es explícito y creíble, los usuarios establecen un compromiso creíble en un sentido motivacional, que, según Shepsle (1998), es aquel que se produce cuando no existe divergencia entre los incentivos ex-ante (antes del inicio de la ejecución contractual, es decir, en $t 0$ ) y los incentivos ex-dure (durante la ejecución contractual, es decir, en $t 1$ ). Quien establece el compromiso continúa luego teniendo como primera preferencia respetarlo y esta consistencia intertemporal no es producida exógenamente por una sanción negativa que lo coacciona a seguir un curso de acción orientado hacia el cumplimiento. Considerando esa orientación hacia el cumplimiento, el compromiso es autoejecutable (self-enforcing) y creíble.

En el caso de un individuo con preferencias oportunistas en $t 0$, hay divergencia con las preferencias respecto a las acciones de cumplimiento comprometidas en $t 1$ y en ausencia de coacción externa no cumpliría su compromiso. Así, está comprometido en un sentido coercitivo, porque la mejor alternativa es cumplir, porque no hacerlo sería altamente costoso. En cambio, quien establece un compromiso en sentido motivacional no se desviará en $t 1$ de su curso de acción, aunque tuviera el poder discrecional para hacerlo. En el caso de los usuarios de RNUC interesados en acceder a los instrumentos de política pública, en $t 0$ su primera preferencia es cooperar, es decir, cumplir.

Esto ocurre con los usuarios que aspiran a obtener microcréditos, certificaciones y pagos por servicios ambientales y que tienen derechos de propiedad sobre un sistema de recursos y que gracias a sus reglas comunitarias ya han logrado la acción colectiva. Si, según Ostrom (1995), la utilidad de los usuarios se obtiene en función de los beneficios de extracción y los costos de provisión, entonces, los beneficios derivados del acceso a los instrumentos de política pública señalados se suman a los que ya obtienen producto de la extracción de unidades del recurso. Además, las prestaciones aportan a reducir costos de provisión, por ejemplo, si les permiten financiar el mejoramiento de infraes- 
tructuras, la contratación de servicios de asistencia técnica, etcétera. La expectativa de una utilidad neta más alta hace que los usuarios tengan, por tanto, como primera preferencia en $t 0$ cumplir, y dado que el cumplimiento permite acceder a los beneficios de las prestaciones, está preferencia se mantiene en $t 1$.

También tendrán como primera preferencia cumplir los usuarios interesados en acceder a derechos de propiedad que asigna el Estado. Si en $t 0$ el Estado asigna derechos de propiedad, la expectativa de los usuarios es que si en $t 1$ cumplen con requerimientos de sustentabilidad a través de la acción colectiva, obtendrán los beneficios derivados de acciones de extracción de unidades del sistema de recursos que realizan en $t 1$, porque tendrán derechos de uso sobre ese sistema de recursos. Además, tendrán incentivos para cumplir, considerando que una evaluación positiva del cumplimiento de sus compromisos hará posible la renovación contractual y dispondrán de un período adicional en el que contarán derechos de propiedad sobre un sistema de recursos.

Desde esa perspectiva, con relación a todos los tipos de instrumentos de política pública señalados, para los usuarios que los pretenden su primera preferencia es cumplir los compromisos, con un pago $b+\phi-c$, donde $b$ representan los beneficios derivados de contar con esos instrumentos, $\phi$ representa un parámetro relacionado con la valoración de los beneficios futuros si ex-post (después de finalizada la ejecución contractual, es decir, en $t 2$ ) se renueva el contrato, a lo que se resta $c$, que es el costo (que incluye costos de provisión y otros adicionales) internalizado por cumplir compromisos contractuales (donde $b>c$ ). Considerado esas preferencias de los usuarios, es claro que la acción colectiva es un medio para acceder a las prestaciones estatales, y lograrla implica cumplir los compromisos, porque esos compromisos se refieren al uso sustentable de sus RNUC.

b) Rendición de cuentas ex-dure y ex-post. El segundo atributo de diseño institucional de los instrumentos de política pública señalados es que existen actividades de rendición de cuentas y de monitorización. Según la teoría principal-agente, las monitorizaciones (que incluyen auditorías, informes de actividades, etcétera) son actividades realizadas por el principal (quien delega) orientadas a obtener información sobre actividades comprometidas contractualmente por un agente (el delegado). El propósito es reducir la asimetría de información sobre las acciones del agente, con el fin de disminuir la probabilidad de ocurrencia de comportamientos oportunistas (Miller, 2005).

Desde una perspectiva diferente, respecto a la monitorización, proponemos centrar la atención en la oportunidad que representa para los usuarios de RNUC rendir cuentas en $t 1$ y $t 2$, porque puede evidenciar el cumplimiento de sus compromisos. Tienen buenas razones para hacerlo considerando sus preferencias. Esto es posible gracias al principio de publicidad como un atributo de diseño institucional. Este principio tiene tres dimensiones: conocimiento general, $X$ es público si todos saben $X$ (las partes interesadas tienen acceso a la misma información); conocimiento mutuo, $X$ es público si todos saben $X$ y todos saben que todos saben $X$; debate crítico, la información disponible como producto de las dimensiones anteriores es transparente y debe ser utilizada para 
ser debatida críticamente. Esto permite que la información sobre las acciones y los resultados de los usuarios de RNUC esté disponible para el escrutinio, ya que puede ser inspeccionada, criticada y contestada por los involucrados (Luban, 2003).

c) Renovación condicional como sanción creíble. El diseño institucional de los instrumentos de política pública genera incentivos en los usuarios, afectando su conjunto de oportunidad, porque la continuidad del contrato en un nuevo período es contingente a una evaluación positiva del cumplimiento de compromisos en el período contractual previo. Así se configura el parámetro $\phi$ antes indicado, que hace que los usuarios de RNUC valoren altamente la acción colectiva que emprenden. La renovación contractual es la sanción positiva que incentiva hacia el cumplimiento a los usuarios. La sanción es publicitada en $t 0$ como información que establece que en $t 2$ está comprometida una acción estatal (la renovación contractual como recompensa), que es consecuencia de las acciones realizadas en $t 1$ por los usuarios.

A partir de esa información, los usuarios forman creencias que orientan su acción hacia el cumplimiento porque la sanción positiva es una promesa creíble del Estado. Tienen la expectativa de que habrá coherencia intertemporal del Estado entre su promesa en $t 0$ y su cumplimiento en $t 2$. La promesa de sanción positiva es creíble, porque, siguiendo a Elster (2006), una promesa es creíble si quien la realiza tiene los motivos y los medios para hacerlo. Además, la promesa previa es creíble y exigible porque es autoejecutable y lo es porque, de acuerdo a lo señalado por Schelling (1964), quien la realiza voluntaria y públicamente luego no puede renunciar a hacerlo, está obligado a hacerlo y tiene que seguir con el curso de acción establecido, que es irreversible.

Como síntesis, señalamos que los atributos del diseño institucional de los instrumentos de la política pública afectan la situación de acción operativa, específicamente los costos y beneficios de los usuarios, lo que produce incentivos adicionales para que se impliquen en la acción colectiva.

\section{Conclusiones}

En este artículo analizamos la acción colectiva comunitaria en torno a la gobernanza de RNUC desde un punto de vista diferente. Planteamos que adquiere una doble dimensión cuando los usuarios están interesados en recibir aportes estatales cuyo requisito es que se demuestre un uso sustentable de sus RNUC. Realizamos una explicación teórica sobre esta doble dimensión a partir de los conceptos de situación de acción y de situaciones de acción adyacentes. En primer lugar, centramos la atención en la situación de acción operativa, que es el espacio microsituacional de decisiones que se produce a nivel comunitario en torno a los RNUC.

La acción colectiva se expresa en la realización de forma conjunta de tareas de apropiación y provisión. La situación de acción operativa configurada desde esas tareas tiene la estructura de un juego de seguridad, en el que los usuarios coordinan expectativas y seleccionan un equilibrio cooperativo, porque forman 
la creencia de que todos concurrirán en un esfuerzo compartido, cumpliendo con lo establecido en las reglas comunitarias de apropiación y provisión. De esta forma, los usuarios superan problemas de apropiación y provisión como formas específicas de problemas de acción colectiva. Distribuyen de forma equitativa beneficios (de extracción) y costos (de provisión) de la acción colectiva en las tareas señaladas y evitan la sobreexplotación de sus RNUC. Entonces, en la situación de acción operativa se produce una de las dimensiones de la acción colectiva: permite el uso sustentable en el uso de RNUC.

La segunda dimensión de la acción colectiva se produce desde la adyacencia entre la situación de acción operativa y la estatal. La adyacencia o conexión es fundamentalmente estructural, porque el diseño institucional de instrumentos de política pública, como los pagos por servicios ambientales, las ecocertificaciones y las concesiones de derechos de uso, entre otros, tiene determinados atributos que incentivan a los usuarios para lograr la acción colectiva. La razón es que así cumplirán con el requisito de demostrar el uso sustentable de sus RNUC. De esta forma, los usuarios adicionan los beneficios obtenidos desde la extracción de unidades de un sistema de recursos a los logrados gracias al acceso a los apoyos estatales.

Como indicamos al principio del artículo, analizar la relación entre el Estado y comunidades usuarias de RNUC como una adyacencia de situaciones de acción generada por el diseño institucional de instrumentos de política pública aporta una nueva perspectiva para el estudio de procesos de cogobernanza. Además, amplía el espectro de aplicaciones de los conceptos de situación de acción y situaciones de acción adyacentes para el estudio de la gobernanza policéntrica.

Según Ostrom et al. (2017), los resultados producidos en una situación de acción pueden evaluarse desde criterios como la eficiencia económica, la equidad distributiva (acceso en el largo plazo de comunidades empobrecidas a recursos naturales), la adaptabilidad y la sustentabilidad ambiental. Si diseños institucionales contenidos en instrumentos de política pública pueden aportan a lograr ese tipo de resultados, es necesario, como lo hicimos en nuestra propuesta teórica, analizar los atributos de esos diseños. Lo que se busca analizar es si estamos en presencia de un buen diseño institucional, que, según Goodin (2003) es aquel que orienta hacia el cumplimiento porque motiva a seguir una conducta esperada, la que, por su parte, ayuda a la generación de resultados socialmente valorados.

\section{Referencias bibliográficas}

AnDERSON, Leigh; LOCKER, Laura y NugENT, Rachel (2002). «Microcredit, social capital, and common pool resources». World Development, 30 (1), 95-105. <https://doi.org/10.1016/S0305-750X(01)00096-1>.

Araújo, Marcio; Schneider, Sérgio; Ploeg, Jan van der; Souza, Marcelino de (2016). "The collective action on governing the commons in the sorroundings of protected areas». Ambiente \& Sociedade, 19 (4), 21-38. <http://dx.doi.org/10.1590/1809-4422asoc134199v1942016>. 
Basurto, Xavier, Gelvich, Stefan y Ostrom, Elinor (2013). «The socio-ecological system framework as a knowledge classificatory system for benthic small-scale fisheries». Global Environmental Change, 23 (6), 1366-1380. <https://doi.org/10.1016/j.gloenvcha.2013.08.001>

BOwLES, Samuel (2004). Microeconomics: Behaviour, institutions and evolution. Princeton: Princeton University Press.

CÁRDENAS, Juan y ORTIZ, Juan (2018). «Acción colectiva para abordar conflictos socio-ambientales. El caso de Santurbán». En: HernándeZ, Andrés (comp.). Modos de gobernanza del agua y sostenibilidad. Aportes conceptuales y análisis de experiencias en Colombia. Bogotá: Ediciones Uniandes.

CÁRDENAS, Juan; RodrígueZ, Luz y JOHnSON, Nancy (2011). «Collective action for wathershed management: Filed experiments in Colombia and Kenia». Enviroment and Development Economics, 16 (3), 275-303. <https://doi.org/10.1017/S1355770X10000392>.

Cole, Daniel y Grossman, Peter (2010). «Institutions matter! Why the Herder Problem in not a Prisioner's Dilemma». Theory and Decision, 69 (2), 219-231. $<$ https://doi.org/10.1007/s11238-008-9121-3>.

Cole, Daniel y MCGINNIS, Michael (2017). «Introduction to volumen 3». En: Cole, Daniel y MCGINNIS, Michael (eds.). Elinor Ostrom and the Bloomington School of Political Economy. Volume 3. A framework for policy analysis. Maryland: Lexington Books.

Cox, James; Ostrom, Elinor; SAdiraJ, Vjollca y WALKer, James (2013). «Provision versus Appropriation in Symmetric and Asymmetric Social Dilemmas». Southern Economic Journal, 79 (3), 496-512. <https://doi.org/10.4284/0038-4038-2012.186>.

CruZ, José de la; Ávila, Víctor; Rivera, María y VizCARra, Ivonne (2016). «Protected natural areas and common use system of forest resources in Nevado de Toluca». Revista Mexicana de Ciencias Forestales, 7 (38), 25-42. <https://doi.org/10.29298/rmcf.v7i38.2>.

ElsTER, Jon (2006). «Los secuestros en las guerras civiles». En: MockUs, Antanas y CANTE, Freddy (comps.). Acción colectiva, racionalidad y compromisos previos. Bogotá: Universidad Nacional de Colombia.

Fisher, Brendan; Kulindwa, Kassim; Mwanyoca, Iddi; Turner, Kerry y BurGESS, Neil (2010). «Common pool resource management and PES: Lessons and constraints for water PES in Tanzania». Ecological Economics, 69 (6), 1253-1261. $<$ https://doi.org/10.1016/j.ecolecon.2009.11.008>.

Foley, Paul y MCCAY, Bonnie (2014). «Certifying the commons: Eco-certification, privatization, and collective action». Ecology and Society, 19 (2), 28. <http://dx.doi.org/10.5751/ES-06459-190228>.

GARDNER, Roy; OSTROM, Elinor y WALKER, James (1990). «The nature of common pool resources problem». Rationality and Society, 2, 335-358. <https://doi.org/10.1177/1043463190002003005>.

Goodin, Robert (2003). "Las instituciones y su diseño». En: Goodin, Robert (comp.). Teoría del diseño institucional. Barcelona: Gedisa.

Greiber, T. H. (ed.) (2010). Pagos por servicios ambientales: marcos jurídicos e institucionales. Gland: UICN.

GRITSENKO, Daria (2018). «Explaining choices in energy infrastructure development as a network of adjacent action situations: The case of LNG in the Baltic Sea region». Energy Policy, 112, 74-83. <http://dx.doi.org/10.1016/j.enpol.2017.10.014>. 
GrubY, Rebecca y BASURTO, Xavier (2013). «Multi-level governance for large marine commons: Politics and polycentricity in Palau's protected area network». Environmental Science \& Policy, 33, 260-272. <https://doi.org/10.1016/j.envsci.2013.06.006>.

GUPTA, Krishna (2005). The political economy of forest management: The importance of institutions and social capital. Nueva Delhi: Allied Publisher PVT.

HARDIN, Garret (2009). "The tragedy of the commons». Journal of Natural Resources, 1 (3), 243-253. <https://doi.org/10.1080/19390450903037302>.

Herreros, Francisco (2007). "Confianza y cooperación en ausencia del Estado». Revista Internacional de Sociología, LXV (46), 87-105. <http://dx.doi.org/10.3989/ris.2007.i46.5>.

JANSSEN, Marco (2013). "Governance of common-pool resources». En: SHOGREN, Jason (ed.). Encyclopedia of energy, natural resource, and environmental economics, 3. San Diego (CA): Elsevier.

KERR, John (2014). «Incentives, conditionality and collective action in payment for enviromental services». International Journal of the Commons, 8 (2), 595-616. <http://doi.org/10.18352/ijc.438>.

KIMmiCH, Christian (2013). «Linking action situation: Coordination, conflicts, and evolution in electricity for irrigation in Andhra Pradesh, India». Ecological Economy, 90, 150-158. <https://doi.org/10.1016/j.ecolecon.2013.03.017>.

KolINJIVADI, Vijay et al. (2014); ADAMOWSKI, Jan y KosOY, Nicolás (2014). «Recasting payments for ecosystem services (PES) in water resources management: A novel institutional approach». Ecosystem Services, 10, 144-154. $<$ https://doi.org/10.1016/j.ecoser.2014.08.008>.

LAPUENTE, Víctor (2007). A political economy approach to bureaucracies. Madrid: Instituto Juan March de Estudios e Investigaciones, Centro de Estudios Avanzados en Ciencias Sociales, University of Oxford.

LINARES, Francisco (2015). «Social networks, social norms and worker's resistance: A computational simulation analysis». En: MiCA, Adriana; WinczoreK, Jan y WISNIEWSKI, Rafael (eds.). Sociologies of formality and informality. Frankfurt am Main: Peter Lank Edition.

LubAN, David (2003). «El principio de publicidad». En: Goodin, Robert (comp.). Teoría del diseño institucional. Barcelona: Gedisa.

MCGINNIS, Michael (1999). «Introduction». En: MCGINNIS, Michael (ed.). Polycentricity and local public economies: Readings from the workshop in political theory and policy analysis. Ann Arbor: The University of Michigan Press.

- (2000). «Introduction». En: MCGINNIS, Michael (ed.). Polycentric games and institutions: Readings from the workshop in political theory and policy analysis. Ann Arbor: The University of Michigan Press.

- (2011a). «Networks of adjacent action situations in polycentric governance». The Policy Studies Journal, 39 (1), 51-78. <https://doi.org/10.1111/j.1541-0072.2010.00396.x>.

- (2011b). «Introduction to volume 3». En: ColE, Daniel y MCGInNIS, Michael (eds.). Elinor Ostrom and the Bloomington School of Political Economy. Volume 3. A framework for policy analysis. Maryland: Lexington Books.

- (2017). "The IAD framework in action: Understanding the sources of the design principles in Elinor Ostrom's governing the commons». En: ColE, Daniel y 
MCGINNIS, Michael (eds.). Elinor Ostrom and the Bloomington School of Political Economy. Volume 3. A framework for policy analysis. Maryland: Lexington Books.

MCGINNIS, Michael y OSTROM, Elinor (2014). «Social-ecological system framework: Initial changes and continuing challenges». Ecology and Society, 19 (2), 30. $<$ http://dx.doi.org/10.5751/ES-06387-190230>.

Merino, Leticia y RobSOn, James (2006). El manejo de recursos de uso común: Pago por servicios ambientales. México DF: CSMSS, The Christensen Fund., Fundación Ford, Semarnat, INE.

MilleR, Gary (2005). «Solutions to principal-agent problems in firms». En: MENARD, Claude y SHIRley, Mary (eds.). Handbook of new institutional economics. Dordrecht: Springer.

MurALI, Kallur (2006). «Microfinance, social capital and natural resource management systems: conceptual issues and empirical evidences». International Journal of Agricultural Resources Governance and Ecology, 5 (4), 327-337. $<$ https://doi.org/10.1504/IJARGE.2006.009924>.

Ostrom, Elinor (1990). Governing the commons. The evolution of instituions for collective action. Cambridge Massachusetts: Cambridge University Press.

- (1995). «Self-organization and social capital». Industrial and Corporate Change, 4 (1), 131-159. <https://doi.org/10.1093/icc/4.1.131>.

- (2002). «Common-pool resources and institutions: Toward a revised theory». En: GARDNER, Bruce y RAUSSER, Gordon (eds.). Handbook of agricultural economics. Volume 2 A. Amsterdam: North-Holland.

- (2005). Understanding institutional diversity. Princeton: Princeton University Press.

- (2007). "Collective action theory». En: Bolx, Charles y STOKES, Susan (eds.). The Oxford handbook of comparative politics. Oxford: Oxford University Press.

- (2009). "A general framework for analyzing sustainability of socio-ecological sytems». Science, 325, 419-422. <http://dx.doi.org/10.1126/science.1172133>.

- (2011). "Background on the institutional analysis and development framework». Policy Studies Journal, 39 (1), 7-27. <https://doi.org/10.1111/j.1541-0072.2010.00394.x>.

Ostrom, Elinor y Cox, Michael (2010). «Moving beyond panaceas: A multi-tiered diagnostic approach for social-ecological analysis». Environmental Conservation, 37 (04), 451-463. <https://doi.org/10.1017/S0376892910000834>.

OsTROM, Elinor y GARDNER, Roy (1993). «Coping with asymmetries in the commons: Self-governing irrigation systems can work». Journal of Economics Perspectives, 7 (4). <http://dx.doi.org/10.1257/jep.7.4.93>.

Ostrom, Elinor; GARDNer, Roy y Walker, James (1994). Rules, games \& common-pool resources. Michigan: The University of Michigan Press.

Ostrom, Elinor; Gibson, Clark; ShivaKumar, Sujai y Andersson, Krister (2017). "Appendix A: The institutional analysis and development framework». En: ColE, Daniel y MCGinNIS, Michael (eds.). Elinor Ostrom and the Bloomington School of Political Economy. Volume 3. A framework for policy analysis. Maryland: Lexington Books.

Ostrom, Elinor y Hess, Charlotte (2010). «Private and common property rights». En: BoucKaerT, Boudewijn (ed.). Property law and economics. Northampton (MA): Edgar Elgar. 
Ostrom, Elinor y SCHLAGER, Edella (1996). «The formation of property rights». En: Hanna, Susan; Folke, Carl y MÄLER, Karl (eds.). Rights to nature. Ecological, economics, cultural, and political principles of institutions for the environment. Washington DC: Island Press.

POLSKI, Margaret y OSTROM, Elinor (2017). "An institutional framework for policy analysis and design». En: COLE, Daniel y MCGINNIS, Michael (eds.). Elinor Ostrom and the Bloomington School of Political Economy. Volume 3. A framework for policy analysis. Maryland: Lexington Books.

Poteete, Amy; Janssen, Marco y Ostrom, Elinor (2010). Working-together. Collective action, the commons, and multiple methods in practice. Princeton: Princeton University Press.

RepúbliCA DE Chile (2008). Decreto Supremo n¹34, que aprueba reglamento de la Ley 20.249 que crea el Espacio Costero Marino de Pueblos Originarios. <http:// www.subpesca.cl/portal/615/articles-9548_documento.pdf>.

SCHELling, Thomas (1964). La estrategia del conflicto. Madrid: Tecnos.

SHEPSLE, Kenneth (1998). "Discrecionalidad, instituciones, y el problema del compromiso del gobierno». En: SAIEGH, Sebastián y TOMMASI, Mariano (comps.). La economía politica: racionalidad e instituciones. Buenos Aires: Eudeba.

STRONZA, Amanda (2009). «Commons management and ecotourism: Ethnographic evidence from the Amazon». International Journal of the Commons, 4 (1), 56-77. <http://doi.org/10.18352/ijc.137>. 